\title{
Differential Activation of the Periaqueductal Gray by Mild Anxiogenic Stress at Different Stages of the Estrous Cycle in Female Rats
}

\author{
Adam J Devall' and Thelma A Lovick' \\ 'School of Clinical and Experimental Medicine, College of Medical and Dental Sciences, University of Birmingham, Birmingham, UK
}

\begin{abstract}
The effect of acute exposure to mild anxiogenic stress on cutaneous nociceptive threshold was investigated in female Wistar rats at different stages of the estrous cycle. Baseline tail flick latencies did not change significantly during the cycle. However after brief exposure to vibration stress ( $4 \mathrm{~Hz}$ for $5 \mathrm{~min}$ ), rats in late diestrus, but not at other cycle stages, developed a hyperalgesia (decrease in tail flick latency). Animals in late diestrus revealed a more than fivefold increase in the density of Fos-like immunoreactive nuclei in the dorsolateral, lateral, and ventrolateral columns in the caudal half of the periaqueductal gray matter (PAG). There was no change in the density of Fos-like immunoreactive nuclei in the PAG in rats in estrus and early diestrus, although rats in proestrus showed a smaller (50\%) but significant increase. Rats undergoing withdrawal from a progesterone dosing regimen (5 mg/kg i.p. twice daily for 6 days) designed to mimic the fall in progesterone that occurs naturally during late diestrus, exhibited a stress-induced hyperalgesia that was similar to animals in late diestrus and a significant increase in Fos-positive cells in the PAG. We suggest that falling levels of progesterone during late diestrus may be a predisposing factor for the development of stress-induced hyperalgesia, which is linked to differential activation of descending pain control circuits in the PAG. Similar changes in women, when progesterone levels fall during the late luteal phase of the menstrual cycle, may contribute to the development of premenstrual symptoms that include increased anxiety and hyperalgesia.

Neuropsychopharmacology (20 I0) 35, II74-I I85; doi:I0.1038/npp.2009.222; published online I3 January 2010
\end{abstract}

Keywords: anxiety-induced hyperalgesia; female rats; tail flick reflex; periaqueductal gray matter; estrous cycle; c-fos

\section{INTRODUCTION}

Sex differences in the perception of pain are well documented. However, despite the generally accepted view that females are more sensitive than males (Greenspan et al, 2007), the underlying cause is not clear and indeed, may be multifactorial. In women, one factor that appears to affect the pain experience is the cyclical variations in sex hormones that occur during the menstrual cycle. Women with existing painful disease states consistently report worsening of their pain during the late luteal phase of the cycle (premenstrual period) (Bajaj et al, 2001; Houghton et al, 2002; Powell-Boone et al, 2005). Cycle-related changes in pain threshold have also been reported in normal healthy women (Riley et al, 1999; Fillingim and Ness, 2000), although a more recent study has challenged this finding (Tousignant-Laflamme and Marchand, 2009).

Correspondence: Dr TA Lovick, College of Medical and Dental Sciences, University of Birmingham, Birmingham BI5 2TT, UK, Tel: 44 I2I 4|4 6929, Fax: 44 121 414 6919, E-mail: t.a.lovick@bham.ac.uk Received 9 September 2009; revised 25 November 2009; accepted 30 November 2009
The perception of pain depends not only on the level of excitation of peripheral nociceptors, but also on the level of activity in the endogenous descending control systems that facilitate or inhibit spinal nociceptive processing (Gebhart, 2004). These two components may be influenced differentially during the menstrual cycle. Thus, although cutaneous nociceptive threshold per se did not change during the cycle, the efficacy of diffuse noxious inhibitory controls, which reduce pain sensitivity by engaging endogenous descending inhibitory control, was reduced during the menstrual and luteal phases (Tousignant-Laflamme and Marchand, 2009). The menstrual cycle may also influence the level of descending facilitation of spinal nociceptive processing. It has long been recognized in the clinic that pain worsens when anxiety levels are increased moderately (Taenzer et al, 1986; Lautenbacher et al, 1999; Kain et al, 2000). Extreme anxiety or fear, on the other hand, elicits hypoalgesia (Ford and Finn, 2008). In many women, the premenstrual period is associated with the development of adverse psychological symptoms that include increased anxiety and negative mood changes (Steiner, 1997). Thus, hormone-linked changes in anxiety levels could be a causative factor for producing menstrual cycle-related 
increases in pain sensitivity by influencing the brain's endogenous pain control mechanisms.

In animal studies, the data concerning cyclical changes in pain sensitivity in females is equivocal. Although there are several reports that cutaneous nociceptive thresholds do not change significantly throughout the estrous cycle in rodents (Aloisi et al, 1994; Vinogradova et al, 2003; Borta and Schwarting, 2005; Terner et al, 2005; Devall et al, 2009), many earlier studies did detect cycle-related differences (Frye et al, 1992; Kayser et al, 1996; Martínez-Gómez et al, 1994; Mogil et al, 2000; You et al, 2006). The underlying reason for these inconsistent findings is not clear but may relate to strain differences and/or to differences in the extent to which the testing methodology engaged endogenous descending control systems. Interestingly, in animals in which no cycle-related change in pain threshold was detected, changes in pain sensitivity linked to hormonal status could be detected after exposure of the rats to environmental stimuli that would be expected to engage descending control systems (Devall et al, 2009; Ryan and Maier, 1988; Walf and Frye, 2003). Thus, parallels between gonadal hormone-linked changes in anxiety and nociception in women and female rats can be shown when the experimental conditions are carefully controlled.

The midbrain periaqueductal gray matter (PAG) is a major source of endogenous descending control of spinal nociceptive processing (Gebhart, 2004). It is also involved in integrating anxiety-like emotional behavior (see Lovick (1996) for review). The changing hormonal profile of the female brain during the estrous cycle has been shown to produce differential effects on neuronal excitability in the PAG (Brack and Lovick, 2007). During the late diestrus phase, the fall in progesterone level is associated with upregulation of $\mathrm{GABA}_{\mathrm{A}}$ receptor subunit expression in the PAG (Griffiths and Lovick, 2005a, b; Lovick et al, 2005). New extrasynaptic receptors with the $\alpha 4 \beta 1 \delta$ configuration are expressed on GABAergic interneurons in the PAG and as a consequence, the output neurons become disinhibited, which leads to the circuitry becoming intrinsically more excitable.

If the functional excitability of the PAG circuitry changes at different stages of the estrous cycle, it is possible that the level of descending control and hence nociceptor responsiveness changes too. To investigate the possibility that differential functional activation of the PAG at different stages of the estrous cycle might underlie changes in pain sensitivity, we examined expression of the immediate early gene $c$-fos in neurons in the PAG in brains taken from rats that had undergone nociceptive testing in the setting of mild anxiogenic stress at different stages of their estrous cycle. In addition, we investigated whether artificial manipulation of progesterone levels, designed to mimic the changes that occur naturally during late diestrus, could influence nociceptive thresholds and stress-induced activation of the PAG.

\section{MATERIALS AND METHODS}

\section{Animals}

Experiments were carried out on female Wistar rats with 200-250 g body weight. Animals were housed in pairs or groups of three in an ambient temperature of $21 \pm 1^{\circ} \mathrm{C}$ under a $12 \mathrm{~h}$ light/dark cycle (lights on at 0700 hours) with free access to food and water. In line with UK Home Office guidelines for the care of animals used for research purposes, home cages contained an adventure toy (upturned plastic box with an entrance cutout) and a chewing stick to provide an enriched environment. The rats were transported from the animal holding room to a different room to carry out all procedures (injections, vaginal smears, and so on) and for behavioral testing. All procedures were undertaken in accordance with the UK Animals (Scientific Procedures) Act 1986.

\section{Determination of the Estrous Cycle Phase}

A vaginal smear was taken daily at approximately 0915 hours and stained using a Quick-Diff FIX staining set (Reagena, Takojantie, Toivala, Finland) to establish that the animals were cycling normally (Brack et al, 2006). An additional smear was taken within $2 \mathrm{~h}$ of the final behavioral experiment to check the cycle stage.

\section{Behavioral Testing}

A detailed description of the behavioral testing protocol has been published recently (Devall et al, 2009). In brief, on 3 days animals were habituated to being confined for $30 \mathrm{~min}$ in a Plexiglass restraint tube. On the fourth day, the rats were placed in the tube and the tail flick latency (TFL) in response to radiant heat from a $600 \mathrm{~W}$ projector lamp focused on a $3 \mathrm{~mm}$ diameter area on the blackened underside of the tail was measured. The voltage supplied to the lamp was adjusted so that a tail flick occurred between 4 and $5 \mathrm{~s}$ of switching on the lamp. TFLs were measured at $5 \mathrm{~min}$ intervals over a $20 \mathrm{~min}$ period to establish baseline values. At the end of the control period, the restraining tube with the rat inside was vibrated at $4 \mathrm{~Hz}$ for $5 \mathrm{~min}$ to induce mild anxiogenic stress. On cessation of the vibration, TFLs were measured at 2 min intervals over the next $10 \mathrm{~min}$ and then at $5 \mathrm{~min}$ intervals for the next $15 \mathrm{~min}$. The next day, when the rats had progressed to a different stage of their cycle, they underwent a second behavioral testing experiment identical to the first one. Control animals underwent the same habituation and testing procedures except that vibration stress was omitted. In control animals, TFLs were consistent throughout the $45 \mathrm{~min}$ experimental period indicating that repeated heating of the tail did not cause tissue damage.

\section{Progesterone Withdrawal Regimen}

The progesterone dosing regimen was based on that described previously (Griffiths and Lovick, 2005a). Female animals received twice daily injections (at approximately 0800 and 1600 hours) of progesterone $(5 \mathrm{mg} / \mathrm{kg}$ i.p. in corn oil; Sigma, Poole, Dorset, UK) for 6 days. On the afternoon of day 6, the progesterone withdrawal group (PWD) received vehicle (corn oil, $0.1 \mathrm{ml}$ per $100 \mathrm{~g}$ i.p.) and again at 0800 hours on day 7 . The high progesterone (HP) group was maintained on progesterone dosing for 7 days. Behavioral testing was carried out on the afternoon of day 7. Control animals in both the HP and PWD groups 
underwent the habituation and experimental testing procedures but the vibration stress was omitted. The dose of progesterone was chosen to maintain plasma levels of progesterone at the high end of the physiological range (Butcher et al, 1975; Doornbos et al, 2009). This dosing regimen disrupted the characteristic daily change in the cytological appearance of vaginal smears seen in spontaneously cycling animals (Figure 1a-d). Progesterone dosing was started on Mondays regardless of the cycle stage of the rat. Within 2-3 days the smears took on an appearance similar to rats in early diestrus (Figure 1e), when progesterone levels would have been elevated for a $24 \mathrm{~h}$ period (Butcher et al, 1975). On withdrawal from progesterone, the cycle appeared to restart and smears taken $24 \mathrm{~h}$
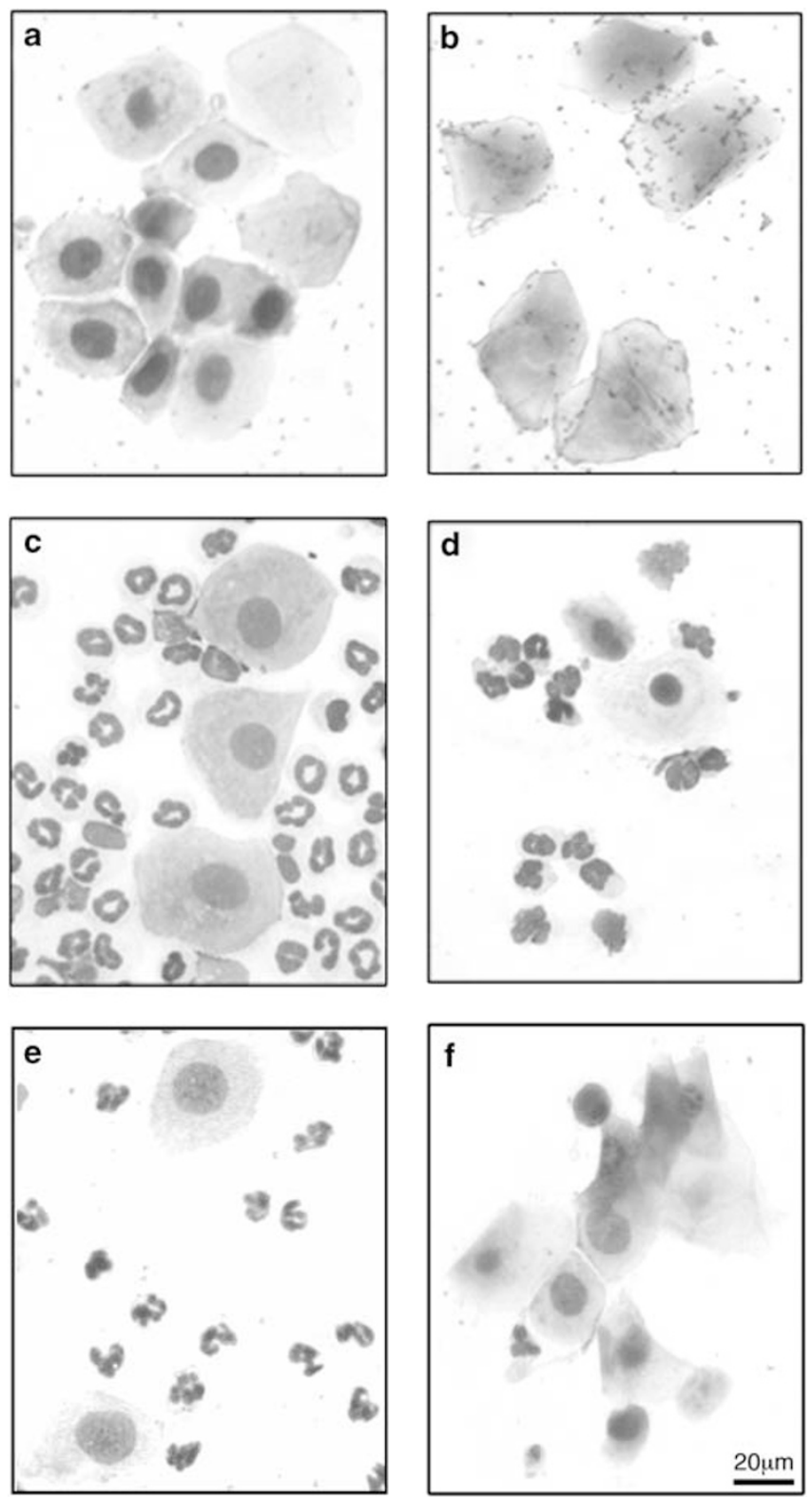

Figure I (a-d) Characteristic cytological appearance of vaginal smears from spontaneously cycling rats. (a) Proestrus, (b) estrus, (c) early diestrus, (d) late diestrus, and (e) smear from a rat after 7 days of progesterone treatment $(5 \mathrm{mg} / \mathrm{kg}$ i.p. twice daily). (f) Smear taken $24 \mathrm{~h}$ after withdrawal from progesterone. after the final injection showed similarities to rats in proestrus (Figure 1f).

\section{Naive Rats}

To determine whether the nociceptive testing regimen and/ or confinement in the restraint tube induced functional activation of the PAG, we handled a group of spontaneously cycling rats daily to obtain vaginal smears but did not undergo any behavioral testing procedures.

\section{Fixation and Histochemical Processing}

Two hours after vibration stress, when acute stimulationinduced activation of $c$-fos has been shown to be optimal (Williams et al, 1990), or at an equivalent time in control (nonstressed) animals, the rats were anesthetized with urethane $(0.5 \mathrm{ml}$ per $100 \mathrm{~g}, 20 \%$ solution i.p.) and perfused retrogradely through the descending aorta with $100 \mathrm{ml}$ heparinized saline $(10 \mathrm{U} / \mathrm{ml})$ followed by $200 \mathrm{ml} 4 \%$ paraformaldehyde in $0.1 \mathrm{M}$ phosphate buffer, $\mathrm{pH}$ 7.4. The brain was removed, post-fixed for $2 \mathrm{~h}$, and transferred to $30 \%$ sucrose in $0.1 \mathrm{M}$ phosphate buffer at $4^{\circ} \mathrm{C}$ for cryoprotection. Naive animals (see above) were anesthetized and perfused at a similar time of day.

Frozen $40-\mu \mathrm{m}$-thick coronal midbrain sections were washed three times in immunobuffer (IB: phosphatebuffered saline containing $10 \mathrm{mM}$ Trizma base and $0.003 \%$ Triton X-100). Hereafter the sections were gently agitated by placing the vials on an orbital shaking platform. Endogenous peroxidase activity was blocked by incubation in hydrogen peroxide solution $(0.5 \%$, in IB containing $1 \%$ normal horse serum; Gibco, UK) for $60 \mathrm{~min}$ at room temperature. The sections were then incubated in anti-Fos antibody (SC-52, 1:5000 in IB containing 10\% normal horse serum; Santa Cruz Biotechnology, CA, USA) at $4{ }^{\circ} \mathrm{C}$ for $48 \mathrm{~h}$ and washed three times in phosphate-buffered saline containing $10 \mathrm{mM}$ Trizma base (TPBS) before incubation in secondary antibody (711-065-152, 1:500 in IB containing $1 \%$ normal horse serum; Jackson ImmunoResearch, PA, USA) at $4^{\circ} \mathrm{C}$ for $24 \mathrm{~h}$.

The sections were next washed in TPBS and incubated with Extravidin ${ }^{\mathrm{TM}}$-peroxidase $(1: 1500$ in IB; Sigma) for $4 \mathrm{~h}$. After further washes in TPBS, sections were incubated with a nickel-diaminobenzidine solution $(0.04 \%$ nickel ammonium sulfate and $0.01 \%$ diaminobenzidine containing $0.004 \%$ ammonium chloride and $0.2 \%$ glucose in $0.1 \mathrm{M}$ phosphate buffer) for $10 \mathrm{~min}$. An equal volume of this solution containing $2 \mu \mathrm{l} / \mathrm{ml}$ glucose oxidase was then added to supply hydrogen peroxide to the peroxidase-diaminobenzidine precipitation reaction. The progress of the ensuing reaction was monitored by periodic inspection of sections under a microscope and stopped when Fos-positive nuclei could clearly be observed in the tissue but before nonspecific background stain developed, by flooding with excess TPBS. After further washes in TPBS, sections were mounted onto gelatinized slides, dried in air, dehydrated through an ascending series of alcohols, cleared in Histoclear (National Diagnostics, Atlanta, GA, USA), and mounted using Histomount (National Diagnostics). All reagents were obtained from Sigma unless otherwise stated. 


\section{Quantification of Fos-Like Immunostaining}

In an attempt to minimize the effect of batch variations in immunostaining, material from the brains of three rats, usually from different estrous cycle stages or treatment groups, were processed in parallel using the same solutions. For each animal, four sections representative of anteroposterior levels $-6.04,-7.04,-7.8$, and -8.72 (Paxinos and Watson, 2004), hereafter termed levels I-IV, respectively (see Figure 2), were selected for detailed quantitative
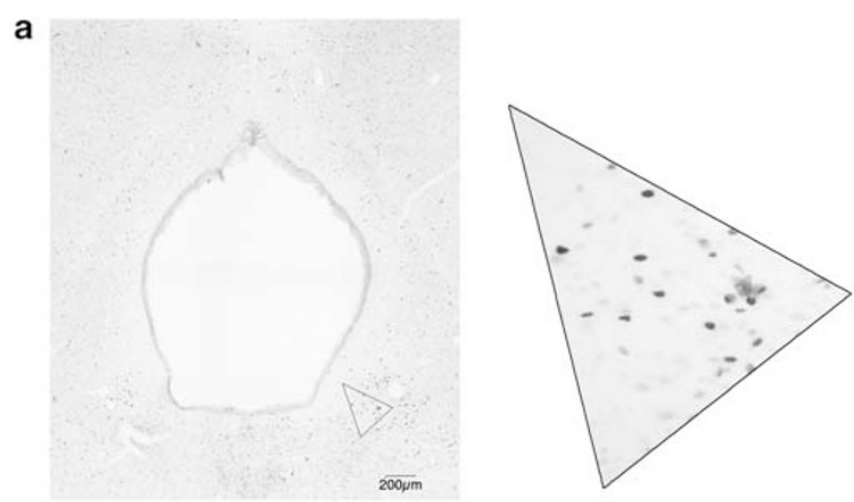

b
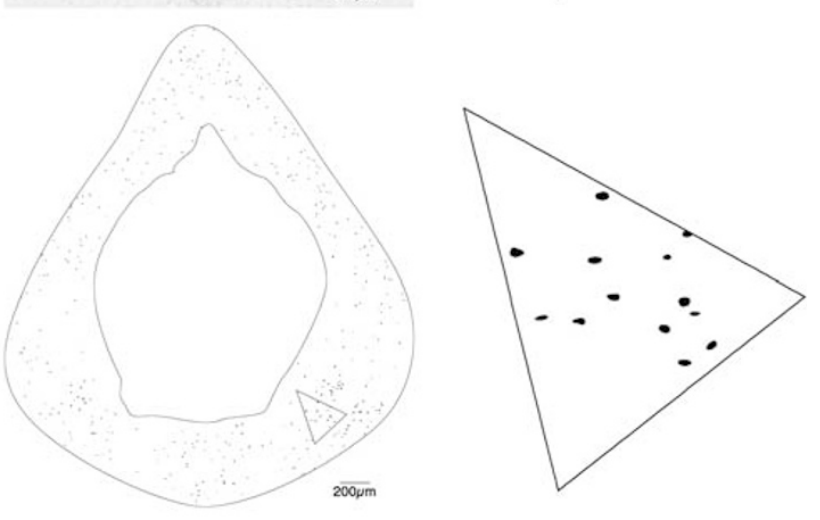

C

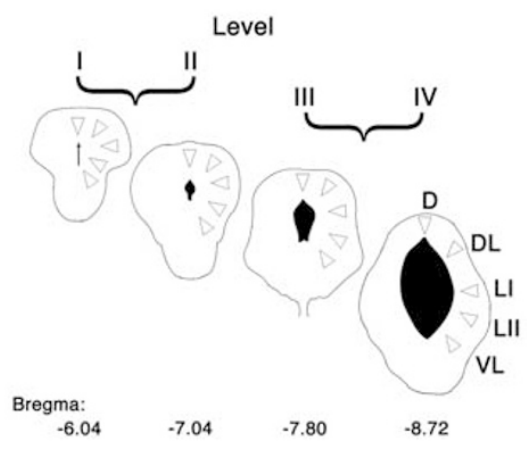

Figure 2 Schematic representation of method used to quantify Fos-like immunoreactive profiles in the periaqueductal gray matter (PAG). (a) Lowpower photomicrograph of a section through the caudal PAG. Highmagnification inset shows individual stained nuclei. (b) Computer-generated plot of all immunostained nuclei recognized in this section. (c) Outline diagrams of sections at the four levels (I-IV) of the PAG sampled. To calculate the density of Fos-positive nuclei, we orientated triangular counting frames representing an area of $60000 \mu \mathrm{m}^{2}$ in the tissue over different columns of the PAG on the computer-generated images of representative sections from each rostrocaudal level. Numbers below sections indicate distance caudal to bregma. D, dorsal; DL, dorsolateral; LI, lateral (I); LII, lateral (II); and VL, ventrolateral. analysis of the density of Fos-immunoreactive nuclei. The analysis was carried out by an experimenter who was blinded both to the experimental procedure and to the hormonal status of the rats. The sections were first photographed at low power $(\times 4$ objective $)$ using phasecontrast microscopy, which allowed the borders of the PAG to be readily delineated. Bright-field images (6-9 fields of view per section) covering the whole PAG were then captured using the $\times 10$ objective and combined electronically to create a photomontage using image analysis software (LUCIA G, v4.2.8; Laboratory Imaging, Czech Republic). The phase-contrast image was scaled to match the magnification of the bright-field image and overlaid at $50 \%$ opacity using imaging software (Adobe Photoshop CS, San Jose, CA, USA). The outlines of the PAG and aqueduct were then delineated using a drawing tablet (Wacom, Krefeld, Germany) and a mask was created over the image so that only the PAG remained visible.

Image analysis software (ImageJ, v1.40; National Institutes of Health, USA) was used to identify Fos-positive nuclei. A threshold was set that excluded all pixels except those that were within the darkest $2.5 \%$ of the image. The resulting mask was analyzed using Imagej's analyze particles function, filtering out those particles that had a size $<15$ or $>100$ pixels $^{2}$ (equating to an area of $<14.8$ and $>98.8 \mu \mathrm{m}^{2}$ ) and a circular morphology with the ratio of the shortest to longest axis of $\leqslant 0.65: 1$. On the basis of initial comparisons between computer-generated plots and manual plotting of stained nuclei using a drawing tube, these factors described a particle that was clearly distinguishable from the background and with sufficient circularity and size restrictions to equate to our expectation of PAG nuclear morphology. After this automated step, a map of the section showing the location of all identified Fos-positive nuclei within the boundaries of the PAG was generated. For each section, the PAG was subdivided into its longitudinal columns as described in the rat brain atlas of Paxinos and Watson (2004): dorsal (D), dorsolateral (DL), lateral (subdivided into L1 and LII), and ventrolateral (VL) (Figure 2). To sample the density of immunolabeled cells within each column of the PAG, we orientated a triangular counting frame representing an area of $60000 \mu \mathrm{m}^{2}$ within the tissue over the image with the apex pointing toward the aqueduct (Figure 2). The number of Fos-positive nuclei present within each frame was then counted manually.

\section{Data Analysis}

All statistical analysis was performed on the raw data using Prism software (v4.0; GraphPad Software, La Jolla, CA, USA). Tail flick data both within and between groups were analyzed using a post hoc Dunnett's test in comparison to appropriate control groups after significant $(p<0.05)$ repeated measures one-way ANOVA. For illustrative purposes data are presented as a percentage of mean baseline TFL, although the level of statistical significance shown on the graphs relates to the raw data. Immunostained cell density in the PAG was analyzed using post hoc Dunnett's and Bonferroni comparisons within appropriate groups after significant $(p<0.05)$ one-way ANOVA. 


\section{RESULTS}

\section{Effect of Estrous Cycle on Vibration Stress-Evoked} Changes in Nociceptive Threshold and Expression of Fos-Like Immunoreactivity in the PAG

The animals used in this study were exposed to nociceptive testing on 2 consecutive days when the rats were in different stages of their estrous cycle. A detailed report of the effects of short lasting vibration stress on nociceptive thresholds on day 1 has been published recently (Devall et al, 2009). In brief, exposure to vibration stress evoked hyperalgesia only when the rats were in the late diestrus phase of their cycle. The new data reported here describe the results of a second study on the same cohort of rats, but carried out the next day, when each animal had progressed to a different stage of the estrous cycle. The behavioral results reported here represent data from those animals in which we carried out a quantitative analysis of Fos expression in the PAG, ie, $n=5-6$ for each group). In these rats, sensitivity to pain was linked to estrous cycle stage, indicating that variability in nociceptor responsiveness within a female population is determined principally by the hormonal profile that characterizes the particular stage of the estrous cycle rather than trait differences between individuals. Moreover, habituation to the testing paradigm on a second exposure, as has been reported for male rats after repeated exposure to a mild anxiogenic stressor (Weinberg et al, 2009; Girotti et al, 2006), did not seem to be present in females.

Baseline TFLs measured during the 20 min control period before onset of the vibration stress were similar in rats at all stages of the estrous cycle and in the animals undergoing different progesterone treatment regimens (Table 1). During exposure to vibration stress, all the animals showed signs of anxiety such as struggling, vocalization, urination, defecation, and lashing of the tail. On cessation of the vibration, the animals rapidly became quiet and tail flick testing was resumed. During the immediate post-stress period, rats in late diestrus displayed a significant hyperalgesia. The mean TFL during the first $10 \mathrm{~min}$ after vibration stress decreased by $16.8 \pm 1.8 \%$ compared to the mean of the TFLs recorded in the $15 \mathrm{~min}$ control period immediately before the onset of vibration $(p<0.01)$. In contrast, there was no significant change in TFL in rats at other stages of the cycle (Figure 3). TFLs did not change significantly over the course of experiments in any of the control animals that were not subjected to vibration stress (Figure 3).

\section{Fos-Like Immunoreactivity in the PAG}

In all animals, we determined the level of functional activation within the PAG resulting from exposure to the nociceptive testing paradigm as reflected by the presence of Fos-like immunoreactive nuclei in brains harvested $2 \mathrm{~h}$ after the delivery of the vibration stress or equivalent time period in nonstressed control animals. For detailed quantitative analysis of Fos-like immunoreactivity in the PAG, we selected animals (5-6 rats per group) in which immunostaining was judged to be optimal.

Fos immunoreactivity in the PAG was visible as rounded immunostained profiles that were present throughout the entire rostrocaudal extent of the nucleus in all the groups of animals used in this study. No Fos-positive profiles were present in sections in which the primary or secondary antibody had been omitted from the staining protocol.

\section{Fos Expression in the PAG in Naive and Control Rats}

A few stained nuclei, which were evenly distributed throughout all sectors of the PAG and at all four rostrocaudal levels sampled, were present in the naive animals. However, the density of immunostained profiles did not differ significantly between rats at different cycle stages (Figure 4). In control animals that underwent nociceptive testing but were not subjected to vibration stress, higher numbers of Fos-positive nuclei were present throughout the PAG at all cycle stages (Figure 4). However, compared to naive rats, the density of labeled cells was significantly higher only in rats in proestrus (Figure 4).

\section{Fos Expression in the PAG After Exposure to Vibration Stress}

When the rats had been exposed to short-lasting vibration stress, the density of labeled cells present in the PAG was increased in late diestrus and to a lesser extent in proestrus (Figure 4). When the data from rats in late diestrus was analyzed in more detail, the elevation in Fos expression was found to be confined to the caudal half of the PAG (levels III and IV, Figure 5) where the density of Fos-immunoreactive nuclei in the dorsolateral, lateral, and ventrolateral columns of the PAG increased by more than $500 \%$ compared to control animals (Figure 5). In contrast, a similar analysis applied to the increase in Fos expression in rats in proestrus revealed that the number of labeled cells increased by less than $50 \%$ compared to the nonstressed control group.

Table I Baseline TFL (Mean \pm SEM) of Three Measurements Taken Before Vibration Stress or at Equivalent Time Points in Control Animals not Subjected to Vibration Stress

Cycle stage/treatment

\begin{tabular}{|c|c|c|c|c|c|c|c|c|c|c|c|c|}
\hline & \multicolumn{2}{|c|}{ Pro } & \multicolumn{2}{|c|}{ E } & \multicolumn{2}{|c|}{ ED } & \multicolumn{2}{|c|}{ LD } & \multicolumn{2}{|c|}{ HP } & \multicolumn{2}{|c|}{ PWD } \\
\hline & Control & Stress & Control & Stress & Control & Stress & Control & Stress & Control & Stress & Control & Stress \\
\hline
\end{tabular}

Abbreviations: Pro: proestrus; E: estrus; ED: early diestrus; LD: late diestrus; HP: high progesterone; PWD: progesterone withdrawal. 
Proestrus

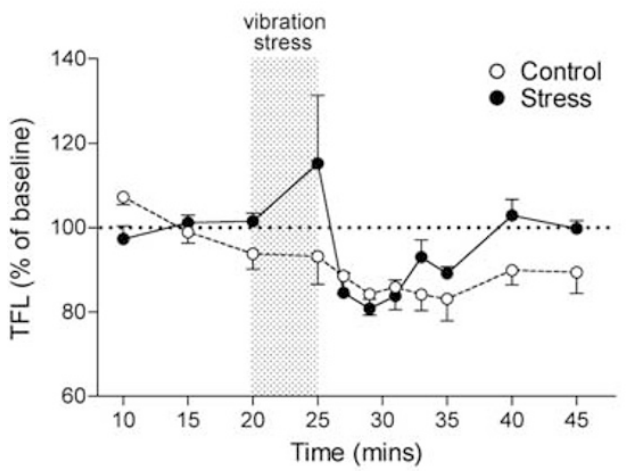

Early Diestrus

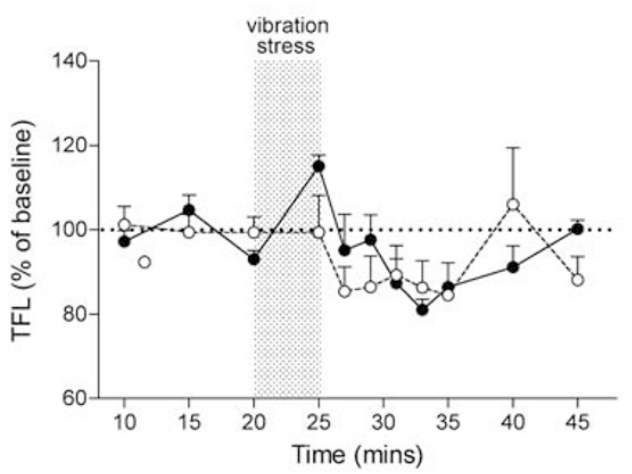

Estrus

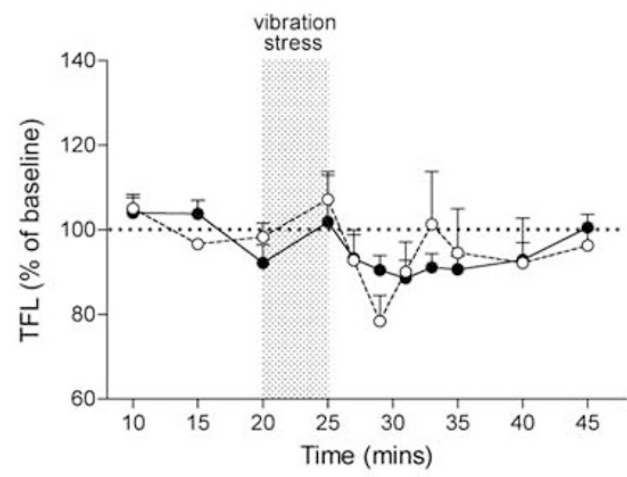

Late Diestrus

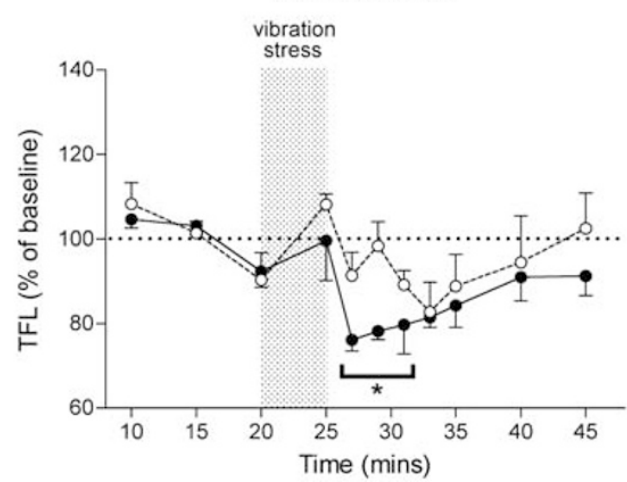

Figure 3 Graph shows time course of the effect of 5 min of vibration stress (gray bar) on tail flick latency (TFL) in female rats at different stages of the estrous cycle. All values (mean \pm SEM) are expressed as a percentage of mean prestress baseline values $(n=5-6$ per group). Control groups were not exposed to vibration stress. $* p<0.05$, post hoc Dunnett's test in comparison to baseline readings after significant $(p<0.05)$ repeated measures one-way ANOVA applied to raw data. $F(8,4,32)=3.531, p=0.0048$.

Moreover unlike rats in late diestrus, the upregulation of Fos-like protein was restricted to the ventrolateral column of the PAG (Figure 6).

\section{Effect of Progesterone Withdrawal}

The baseline TFLs of rats that had been chronically treated with progesterone and were either undergoing withdrawal (PWD, $n=6)$ or maintained on HP $(n=6)$ were similar and did not differ significantly from the spontaneously cycling animals (Table 1). After exposure to the vibration, stressed rats undergoing PWD $(n=6)$ displayed a post-stress hyperalgesia $(16.9 \pm 1.9 \%$ decrease in TFL), which was similar to that seen in spontaneously cycling rats in late diestrus (Figure 7). In contrast, stress did not induce any change in TFL in the HP group. Control animals from the HP and PWD groups that went through the behavioral testing protocol but were not subjected to the vibration stress did not show any change in TFL (Figure 7). Note that to match as far as possible the hormonal profile of progesterone treated rats with those of rats in late diestrus, progesterone-treated rats were killed $24-26 \mathrm{~h}$ after withdrawal or equivalent time for the group maintained on HP. Thus, only one behavioral testing experiment was carried out in this cohort.

Fos-positive nuclei were present throughout the PAG in control, ie, nonstressed rats in the HP and PWD groups. The density of immunostained profiles was similar to that

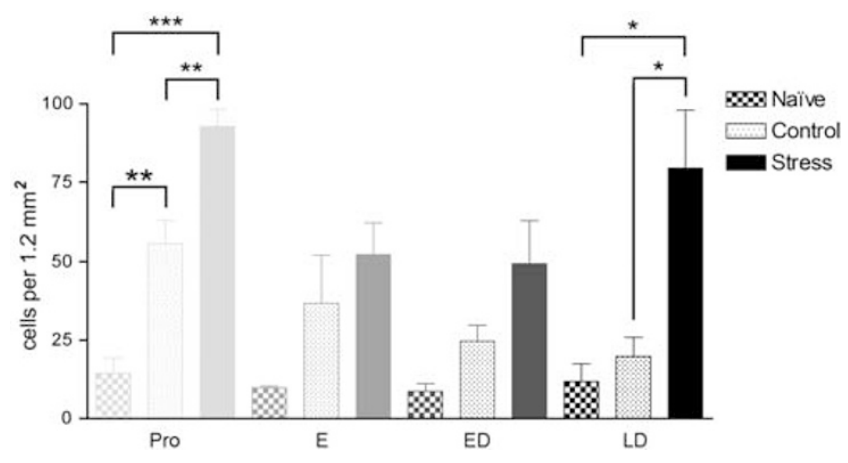

Figure 4 Mean density of Fos-like immunoreactive nuclei in the whole periaqueductal gray matter (PAG) of spontaneously cycling female rats in the stressed and control groups ( $n=5-6$ per group) and in naive rats ( $n=3$ per cycle stage), which had not undergone any behavioral testing procedures. Abbreviations as in Table I. NB Because a priori estrous cycle stage determines responsiveness to stress (see Figure 3), we restricted analysis to within cycle stages. All values mean $\pm \mathrm{SEM}$; $*<0.05$. $* * * 0.0 \mathrm{l}$, ***** $p<0.00$ I, post hoc Bonferroni test after significant $(p<0.05)$ one-way ANOVA within each cycle stage. Proestrus: $F(2,10)=33.430, p<0.0001$; late diestrus: $F(2,10)=7.831, p=0.009$.

seen in spontaneously cycling control rats at different stages of the estrous cycle. After exposure to vibration stress, rats undergoing PWD showed a more than fourfold increase in the mean number of Fos-positive nuclei in the PAG compared to unstressed controls (Figure 8). No change in 
Late diestrus
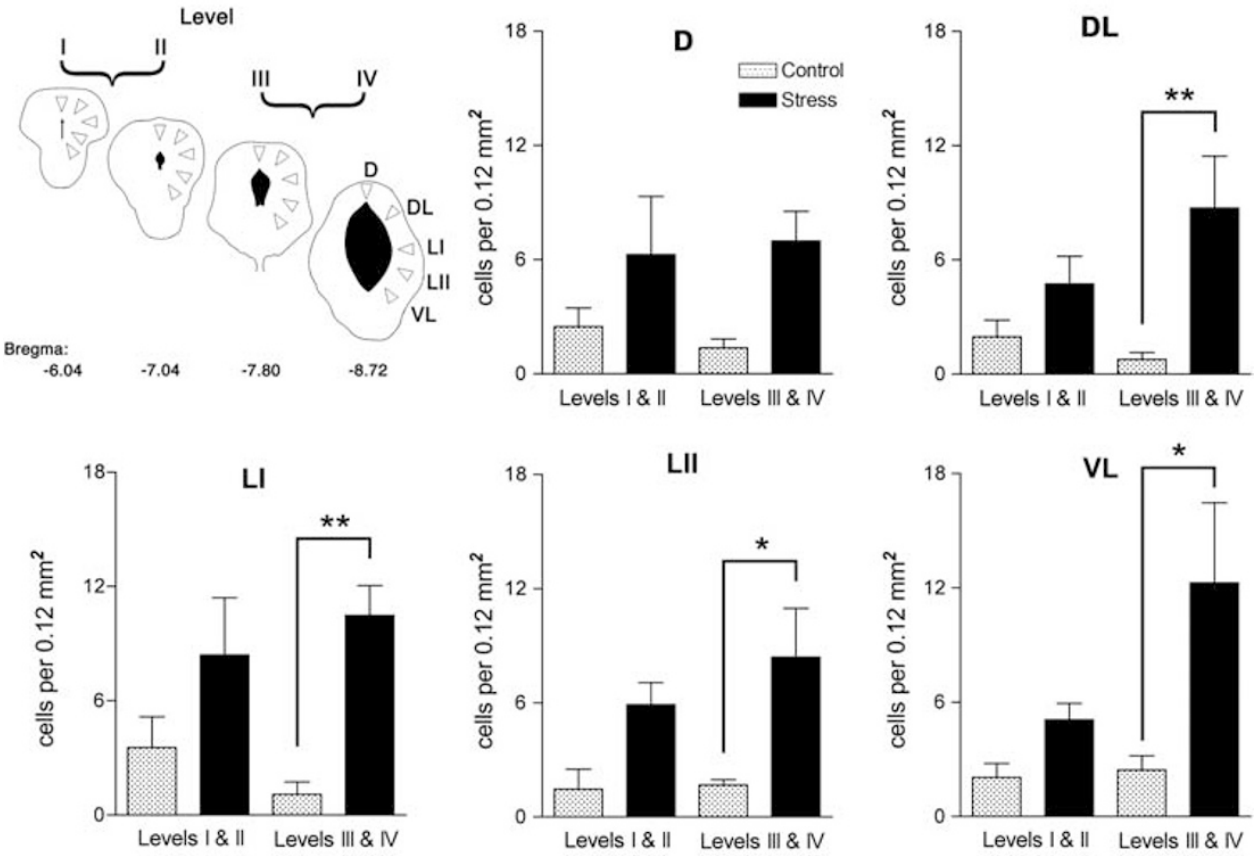

Figure 5 Effect of exposure to 5 min vibration stress on the density of Fos-immunoreactive cells in individual columns of the periaqueductal gray matter (PAG) at rostral (levels I and II) and caudal (levels III and IV) levels in rats in late diestrus. Cartoon depicts areas of PAG sampled (for more details see Figure 2). Histograms show mean Fos immunoreactive cell density indifferent columns of the PAG. All values mean \pm SEM; $* p<0.05$, *** $<<0.01$, post hoc Bonferroni test in comparison to the control group after significant $(p<0.05)$ one-way ANOVA. DL: $F(3,16)=4.772, p=0.015 ; L I: F(3,16)=5.137$, $p=0.01$ I; LII: $F(3,16)=5.030, p=0.012 ; \mathrm{VL}: F(3,16)=4.561, p=0.017$.
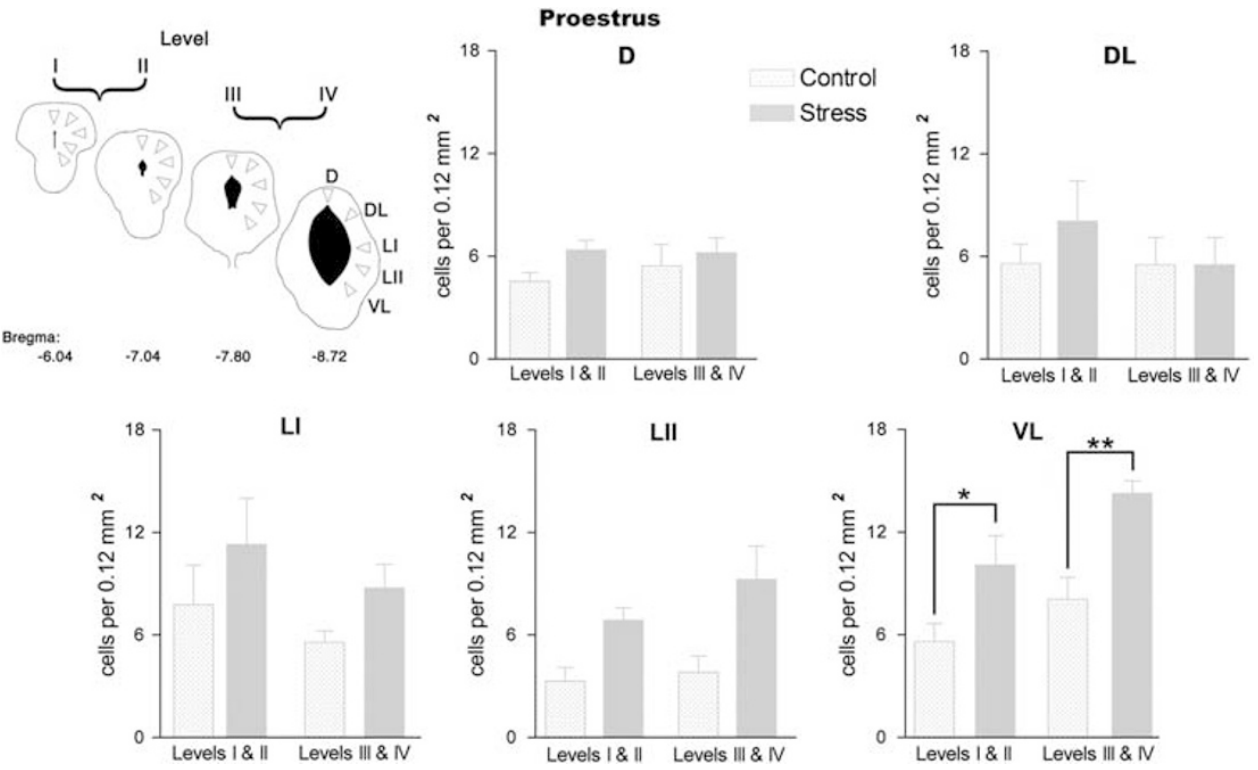

Figure 6 Effect of exposure to 5 min vibration stress on the density of Fos-immunoreactive cells in individual columns of the periaqueductal gray matter (PAG) at rostral (levels I and II) and caudal (levels III and IV) levels in rats in proestrus. Cartoon depicts areas of PAG sampled (for more details see Figure 2). Histograms show mean Fos immunoreactive cell density in different regions of the PAG. All values mean $\pm \mathrm{SEM}$; $* p<0.05$, *** $<<0.0$ l, post hoc Bonferroni test in comparison to the control group after significant $(p<0.05)$ one-way ANOVA. VL: $F(3,16)=8.454, p=0.00$ l.

Fos expression was apparent in the group maintained on HP. When the location of the labeled cells was examined more closely, the increase in density of Fos-immunoreactive nuclei in the PWD group was found to be localized largely in the lateral and ventrolateral columns of the caudal PAG, although a significant increase in labeled cells was also seen in the dorsal column in the rostral half of the PAG (Figure 9). 

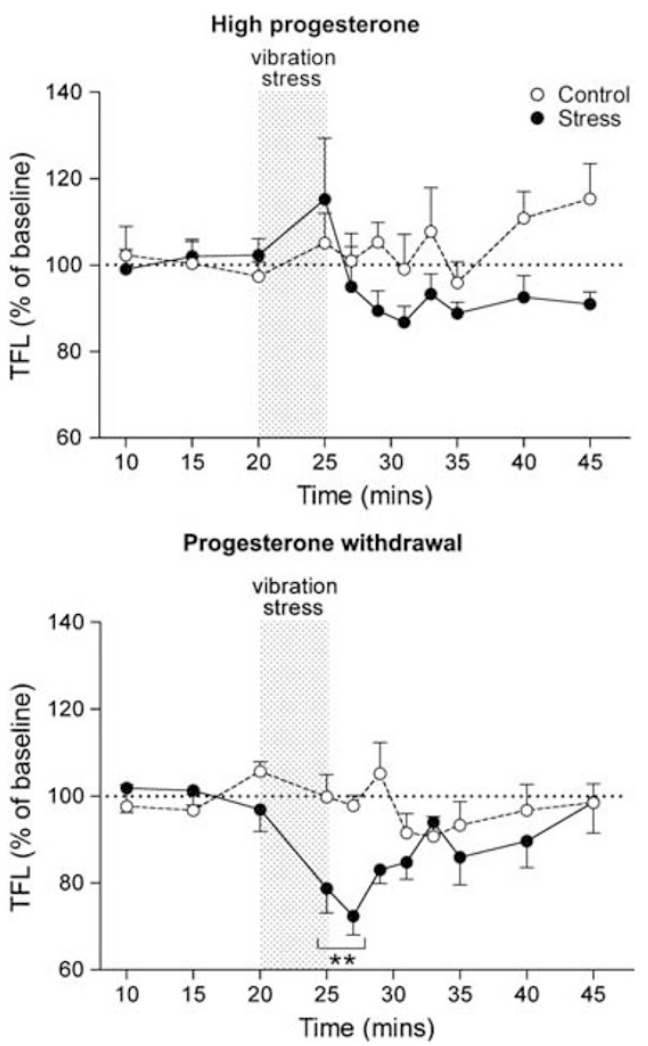

Figure 7 Graph shows time course of the effect of 5 min of vibration stress (gray bar) on tail flick latency (TFL) in female rats that had undergone 7 days treatment with progesterone (HP) or 6 days treatment and $24 \mathrm{~h}$ progesterone withdrawal (PWD). Control groups were not exposed to vibration stress. All values (mean \pm SEM) are expressed as a percentage of mean prestress baseline values ( $n=5-6$ per group). $* * * 0.01$, post hoc Dunnett's test in comparison to baseline readings after significant $(p<0.05)$ repeated measures one-way ANOVA applied to raw data. $F(8,5,40)=4.044, p=0.001$.

\section{DISCUSSION}

In this study in female rats, brief exposure to a mild anxiogenic stressor produced a decrease in nociceptive threshold in rats in the late diestrus phase of their estrous cycle, but not at other stages. This finding contrasts with the results of many previous studies using more intense stressors, which evoked hypoalgesia, and concurs with the consensus view that although fear evoked by intense stress inhibits pain, anxiety evoked by milder stimuli enhances it (see Rhudy and Meagher (2000) and Ford and Finn (2008) for discussions on this point). In our study exposure to mild stress also evoked hyperalgesia in rats undergoing withdrawal from long-term dosing with exogenous progesterone, suggesting that the fall in levels of progesterone that occurs naturally during late diestrus (Butcher et al, 1975) may be the underlying trigger. The appearance of stressinduced hyperalgesia correlated with a four- to fivefold increase in the number of Fos-immunoreactive nuclei in the caudal half of the PAG in rats in late diestrus as well as in the PWD group. During late diestrus, the PAG circuitry becomes intrinsically more excitable due to a reduction in ongoing GABAergic tone, which is linked to upregulation of $\alpha 4, \beta 1$, and $\delta \mathrm{GABA}_{\mathrm{A}}$ receptor subunits on GABAergic

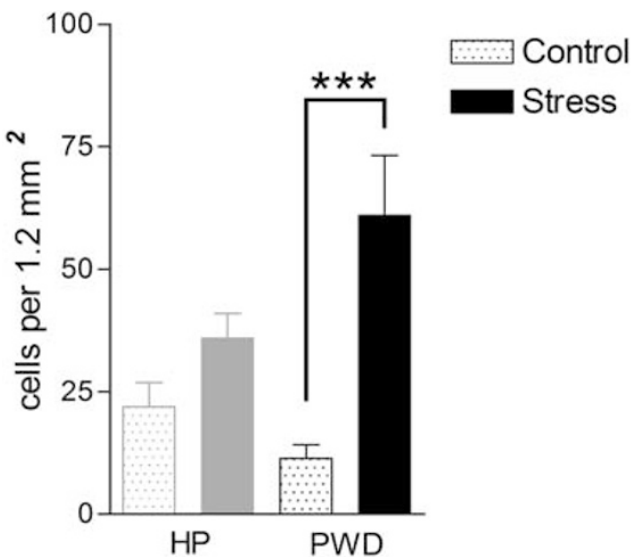

Figure 8 Mean density of Fos-like immunoreactive nuclei in the whole periaqueductal gray matter (PAG) of rats in the stressed and control groups undergoing progesterone treatments ( $n=6$ per group) Abbreviations as in Table I. All values mean \pm SEM; $* p<0.05$. ** $p<0.0$ I, ***** $p<0.00$ I, post hoc Bonferroni test after significant $(p<0.05)$ one-way ANOVA. $F(3,19)=8.700, p=0.008$.

neurons (Brack and Lovick 2007; Lovick et al, 2005; Griffiths and Lovick 2005a). These neuronal changes are linked with the fall in brain levels of progesterone and hence allopregnanolone, the major neuroactive metabolite of progesterone (Butcher et al, 1975; Purdy et al, 1990). Withdrawal from dosing with exogenous progesterone also evoked upregulation of $\alpha 4, \beta 1$, and $\delta \mathrm{GABA}_{\mathrm{A}}$ receptor subunits (Griffiths and Lovick, 2005b), suggesting that the natural fall in progesterone levels that occurs during late diestrus is the trigger for the functional changes within the circuitry in the PAG. It is worth pointing out that to match, as far as possible, the hormonal profile of progesteronetreated rats with those in late diestrus, only one behavioral testing experiment was possible in the former cohort, after $24 \mathrm{~h}$ of progesterone withdrawal. We compared the data from this group to data from spontaneously cycling rats after their second behavioral test. However, because we previously found that the behavioral response of individual spontaneously cycling rats was linked to their estrous cycle stage and not whether they were tested for the first or second time (Devall et al, 2009), we considered this comparison to be valid.

Interestingly, although Fos expression was increased in late diestrus and during progesterone withdrawal, there were subtle differences in the distribution of labeled cells within the PAG in the two conditions. In rats in late diestrus, the increase in expression of Fos protein was confined to dorsolateral, lateral, and ventrolateral columns in the caudal half of the PAG, whereas in rats undergoing progesterone withdrawal, there was an increase in labeled cell numbers in the rostral (dorsal, lateral, and ventrolateral columns) as well as the caudal PAG (lateral and ventrolateral columns). In contrast, the dorsomedial PAG showed only moderate activation. It has been suggested that the dorsomedial PAG is a key neural structure for the selection of the most appropriate behavioral response in a specific dangerous context (Kincheski and Carobrez, 2009). Because the brief, mild vibration stress used in this study is unlikely to have been perceived by the rat as a severe anxiogenic 

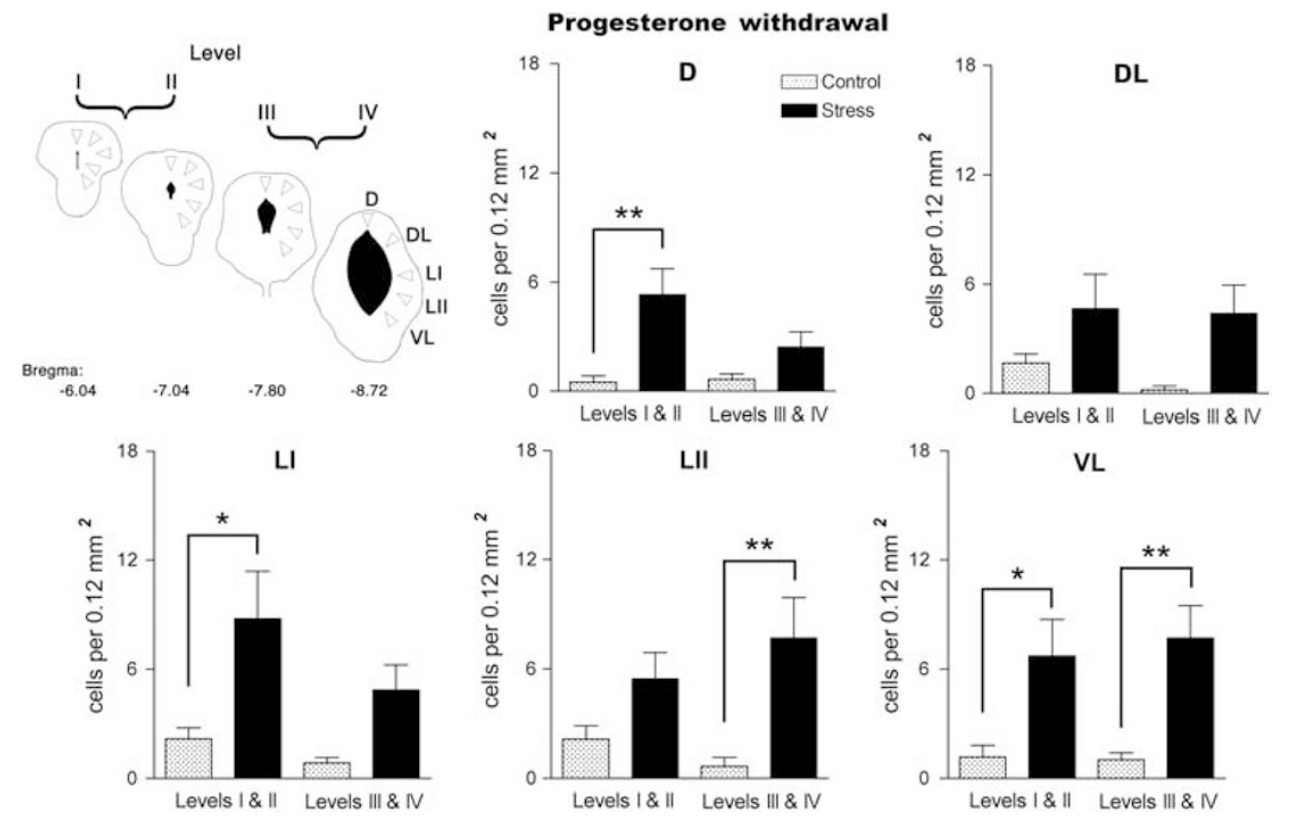

Figure 9 Effect of exposure to 5 min vibration stress on the density of Fos-immunoreactive cells in individual columns of the periaqueductal gray matter (PAG) at rostral (levels I and II) and caudal (levels III and IV) levels in rats undergoing withdrawal from progesterone. Cartoon depicts areas of PAG sampled (for more details see Figure 2). Histograms show mean Fos immunoreactive cell density in different regions of the PAG. All values mean \pm SEM; * $p<0.05$, ** $p<0.01$, post hoc Bonferroni test in comparison to the control group after significant $(p<0.05)$ one-way ANOVA. DL: F $(3,20)=6.726, p=0.0026$; LI: $F(3,20)=5.437, p=0.007 ;$ LII: $F(3,20)=5.18 I, p=0.082 ; V L: F(3,20)=6.5 I 5, p=0.003$.

challenge, the minimal activation of the dorsomedial PAG seen in this study is not unexpected.

The reason for the differences in expression of Fos during late diestrus and during PWD, when the behavioral manifestation of the response to stress in the two groups of rats, ie increased pain sensitivity was indistinguishable, is not clear. Previous studies have shown that physical stressors that are perceived as threatening and evoke confrontational (active) coping strategies are associated with increased Fos expression in rostral, dorsal half of the PAG whereas passive coping leads to preferential activation in the caudal ventrolateral PAG (Keay and Bandler, 2001). The spontaneously cycling rats in this study had already been subjected to the vibration stress and nociceptive testing protocol on the previous day (Devall et al, 2009), whereas rats undergoing progesterone withdrawal were experiencing the stress for the first time. When rats were in late diestrus the behavioral outcome of the exposure to stress was the same, ie, hyperalgesia, regardless of whether the rats were experiencing the stress for the first or second time. However, there may have been subtle differences in the way the stress was perceived, eg, potentially escapable (first exposure) or, as a result of experience, inescapable (second exposure). Because rats undergoing progesterone withdrawal were exposed to stress only once, their perception of the stress may have differed from the rats in late diestrus, leading to a different pattern of activation within the PAG. Activation of structures in the rostral PAG in the PWD group may therefore be associated with an active coping strategy on first exposure to the vibration stress.

The PAG is a source of descending facilitatory and inhibitory pathways to the spinal cord that exert parallel but opposing influences on transmission of noxious input through the dorsal horn (Gebhart, 2004). Descending projections from the PAG relay on ON and OFF cells in the rostral ventromedial medulla that, respectively, facilitate and inhibit nociceptive transmission by their projections to the spinal dorsal horn (Morgan et al, 2008). Both the inhibitory and facilitatory systems are thought to be tonically active, although it has been suggested that under normal, ie nonstressed conditions, a small net facilitatory influence predominates, at least in males (Bee and Dickenson, 2008). During late diestrus, the level of ongoing GABAergic inhibition within the PAG decreases, rendering the circuitry intrinsically more excitable (Brack and Lovick, 2007). The presence of a post-stress hyperalgesia in rats in late diestrus suggests that exposure to the vibration stress may have further tipped the balance between the levels of activity in these descending pathways in favor of facilitation. Such an effect could be produced by an increase in tonic descending facilitation and/or a decrease in tonic descending inhibition. Identification of the phenotype of the Fos-immunoreactive cell population that was upregulated in response to stress will, however, be needed to resolve this issue.

Another possibility is that the increase in functional activation of the PAG in response to brief exposure to stress during late diestrus could reflect a stress-induced increase in afferent input to the PAG. During the initial assessment phase, determination of the emotional significance of a novel and hence inherently stressful stimulus is likely to occur in cortical and/or limbic regions, which then engage the PAG downstream to initiate and integrate the somatomotor and autonomic components of the animal's response. It is well established that mild psychogenic stress activates neurons in various cortical areas that project to the PAG (Beitz 1982; Ceccatelli et al, 1989; Cullinan et al, 1995; 
da Costa Gomez and Behbehani, 1995; Figueiredo et al, 2002; Girotti et al, 2006; Salchner and Singewald, 2002; Gerrits et al, 2003; Rizvi et al, 1991; Rocher et al, 2004; Brown et al, 2005; Floyd et al, 2000; Keay and Bandler, 2001). In particular, activation of the orbitofrontal cortex has been shown to induce biphasic changes in nociceptive threshold: hypoalgesia followed by a delayed hyperalgesia, and both effects could be blocked by inhibiting transmission through the ventrolateral PAG (Zhang et al, 1997). These findings suggest that there may be two functionally distinct pro- and antinociceptive descending pathways from the ventrolateral orbitofrontal cortex to the PAG, which may be activated differentially according to the way the stimulus is perceived by the individual. In support of this idea, studies in male rats have shown that although exposure to a non-noxious stress induced hyperalgesia (Vidal and Jacob, 1982; Jørum, 1988), a more severe stress induced an increase in pain threshold (Rizzi et al, 2001; Vendruscolo et $a l, 2004)$. The mild stressor used in this study would therefore be expected to activate the pro-nociceptive pathways from the PAG. Interestingly, in females stress-induced expression of $c$-fos mRNA in cingulate, frontal, and piriform cortices was estrous cycle dependent, being higher in diestrus compared to proestrus (Figueiredo et al, 2002). Thus, enhanced frontal cortical input to the PAG, combined with an increase in intrinsic excitability of its circuitry, may have been responsible for the enhanced level of functional activation of the PAG by mild stress during the diestrus phase seen in this study.

An unexpected finding in this study was the increase in the density of Fos-labeled cells in the PAG seen in proestrus, particularly in the control (nonstressed) group. Baseline nociceptive thresholds were similar at all cycle stages suggesting that in the absence of stress, there was no change in nociceptive sensitivity in different cycle stages. In any case, it seems unlikely that the brief exposure to the noxious heat stimulus during tail flick testing (less than $60 \mathrm{~s}$ total duration over the $45 \mathrm{~min}$ experiment) would have been sufficient to induce a detectable increase in expression of Fos protein.

All the rats used in our study, including those in proestrus, had been habituated to being confined in the tube over three sessions before the experiment and showed no observable behavioral signs of distress. In this study the density of Fos-positive nuclei in control rats in estrus, early diestrus, or late diestrus that had been habituated to being confined in the restraint tube, but not vibrated, was not significantly different to naive animals. In contrast, rats in proestrus showed a significantly raised expression of Fos protein. There were no overt signs that rats in proestrus were reacting differently to rats in other stages of the cycle. However, we cannot exclude the possibility that rats in proestrus perceived being confined in a different way to animals in other cycle stages and that this led to functional activation within the PAG. Plasma and hence brain levels of estrogen are high in proestrus (Butcher et al, 1975). The presence of estrogen has been shown to amplify stress-induced responses in the prefrontal cortex (Shansky et al, 2004). Because the PAG is driven by afferent input from the PFC (Floyd et al, 2000), exposure of rats to the restraining tube in proestrus, when estrogen levels are high may, even with prior habituation to the apparatus, lead to enhanced activation of the PAG compared to other cycle stages.

During proestrus there is a very brief surge in progesterone production (Butcher et al, 1975). However, the kinetics of this effect are too fast to evoke detectable changes in $\mathrm{GABA}_{\mathrm{A}}$ receptor subunit expression (see Brack and Lovick (2007) for discussion of this point). Nevertheless, exposure of rats to vibration stress during proestrus induced a significant increase in the level of Fos expression in the PAG, albeit some 10-fold lower and much less widespread than the upregulation of the gene expression seen during late diestrus. The trigger for the relatively weak upregulation of $c$-fos in proestrus remains unclear. In any case, in terms of functional outcome it does not appear to have been sufficient to produce a detectable behavioral output related to changes in pain sensitivity.

This study, which was confined to female rats, highlights how neural responsiveness to salient stimuli can be influenced according to the hormonal milieu in which they are received. It would be of interest to compare these findings with data from male rats that have undergone the same stress protocol, as this may shed further light on the functional significance of the patterns of Fos expression that were observed at different stages of the estrous cycle in females.

In summary, we have shown that brief exposure of female rats to a non-noxious anxiogenic stress in late diestrus induced hyperalgesia that was associated with functional activation of neurons in the caudal half of the PAG. We propose that falling concentrations of progesterone in the brain during late diestrus trigger changes in the functional excitability of the descending pro-nociceptive control pathways in the PAG, which renders them more sensitive to activation by mild anxiogenic stress during late diestrus compared to other stages of the estrous cycle. These effects may be directly relevant to the development of premenstrual hyperalgesia in women.

\section{ACKNOWLEDGEMENTS}

This work was supported by MRC Project Grant G070037981940 awarded to TAL. We acknowledge the invaluable technical support provided by Mr Mike Summerfield.

\section{DISCLOSURE}

The authors declare that, except for income received from their primary employer, no financial support or compensation has been received from any individual or corporate entity over the past three years for research or professional service and there are no personal financial holdings that could be perceived as constituting a potential conflict of interest.

\section{REFERENCES}

Aloisi AM, Steenbergen HL, van de Poll NE, Farabollini F (1994). Sex-dependent effects of restraint on nociception and pituitaryadrenal hormones in the rat. Physiol Behav 55: 789-793.

Bajaj P, Arendt-Nielsen L, Bajaj P, Madsen H (2001). Sensory changes during the ovulatory phase of the menstrual cycle in healthy women. Eur J Pain 5: 135-144. 
Bee LA, Dickenson AH (2008). Descending facilitation from the brainstem determines behavioural and neuronal hypersensitivity following nerve injury and efficacy of pregabalin. Pain 140: 209-223.

Beitz AJ (1982). The organization of afferent projections to the midbrain periaqueductal gray of the rat. Neurosci 7: 133-159.

Borta A, Schwarting RK (2005). Inhibitory avoidance, pain reactivity, and plus-maze behavior in Wistar rats with high versus low rearing activity. Physiol Behav 84: 387-396.

Brack KE, Jeffery SMT, Lovick TA (2006). Cardiovascular and respiratory responses to a panicogenic agent in anaesthetised female Wistar rats at different stages of the oestrous cycle. Eur J Neurosci 23: 3309-3318.

Brack KE, Lovick TA (2007). Neuronal excitability in the periaqueductal gray matter during the estrous cycle in female Wistar rats. Neurosci 144: 325-335.

Brown SM, Henning S, Wellman CL (2005). Mild, short-term stress alters dendritic morphology in rat medial prefrontal cortex. Cerebral Cortex 15: 1714-1722.

Butcher RL, Collins WE, Fugo NW (1975). Altered secretion of gonadotropins and steroids resulting from delayed ovulation in the rat. Endocrinol 96: 576-586.

Ceccatelli S, Villar MJ, Goldstein M, Hökfelt T (1989). Expression of c-Fos immunoreactivity in transmitter-characterized neurons after stress. Proc Natl Acad Sci USA 86: 9569-9573.

da Costa Gomez TM, Behbehani MM (1995). An electrophysiological characterization of the projection from the central nucleus of the amygdala to the periaqueductal gray of the rat: the role of opioid receptors. Brain Res 689: 21-31.

Cullinan WE, Herman JP, Battaglia DF, Akil H, Watson SJ (1995). Pattern and time course of immediate early gene expression in rat brain following acute stress. Neurosci 64: 477-505.

Devall AJ, Liu Z, Lovick TA (2009). Hyperalgesia in the setting of anxiety: sex differences and effects of the oestrous cycle in Wistar rats. Psychoneuroendocrinology 34: 587-596.

Doornbos B, Fokkema DS, Molhoek M, Tanke MA, Postema F, Korf JA (2009). Abrupt rather than gradual hormonal changes induce postpartum blues-like behavior in rats. Life Sci 84: 69-74.

Figueiredo HF, Dolgas CM, Herman JP (2002). Stress activation of cortex and hippocampus is modulated by sex and stage of estrus. Endocrinology 143: 2534-2540.

Fillingim RB, Ness TJ (2000). Sex-related hormonal influences on pain and analgesic responses. Neurosci Biobehav Rev 24: 485-501.

Floyd NS, Price JL, Ferry AT, Keay KA, Bandler R (2000). Orbitomedial prefrontal cortical projections to distinct longitudinal columns of the periaqueductal gray in the rat. J Comp Neurol 422: 556-578.

Ford GK, Finn DP (2008). Clinical correlates of stress-induced analgesia: evidence from pharmacological studies. Pain 140: 3-7.

Frye CA, Bock BC, Kanarek RB (1992). Hormonal milieu affects tail flick latency in female rats and may be attenuated by access to sucrose. Physiol Behav 52: 699-706.

Gebhart GF (2004). Descending modulation of pain. Neurosci Biobehav Rev 27: 729-737.

Gerrits M, Westenbroek C, Fokkema DS, Jongsma ME, Den Boer JA, Ter Horst GJ (2003). Increased stress vulnerability after a prefrontal cortex lesion in female rats. Brain Res Bull 61: 627-635.

Girotti M, Pace TW, Gaylord RI, Rubin BA, Herman JP, Spencer RL (2006). Habituation to repeated restraint stress is associated with lack of stress-induced c-fos expression in primary sensory processing areas of the rat brain. Neurosci 138: 1067-1081.

Greenspan JD, Craft RM, LeResche L, Arendt-Nielsen L, Berkley KJ, Fillingim RB (2007). Studying sex and gender differences in pain and analgesia: a consensus report. Pain 132(Suppl 1): S26-S45.

Griffiths JL, Lovick TA (2005a). GABAergic neurones in the rat periaqueductal gray matter express alpha4, beta1 and delta
GABAA receptor subunits: plasticity of expression during the estrous cycle. Neurosci 136: 457-466.

Griffiths JL, Lovick TA (2005b). Withdrawal from progesterone increases expression of alpha4, beta1, and delta GABA(A) receptor subunits in neurons in the periaqueductal gray matter in female Wistar rats. J Comp Neurol 486: 89-97.

Houghton LA, Lea R, Jackson N, Whorwell PJ (2002). The menstrual cycle affects rectal sensitivity in patients with irritable bowel syndrome but not healthy volunteers. Gut 50: 471-474.

Jørum E (1988). Analgesia or hyperalgesia following stress correlates with emotional behavior in rats. Pain 32: 341-348.

Kain ZN, Sevarino F, Alexander GM, Pincus S, Mayes LC (2000). Preoperative anxiety and postoperative pain in women undergoing hysterectomy. A repeated-measures design. J Psychosom Res 49: 417-422.

Kayser V, Berkley KJ, Keita H, Gautron M, Guilbaud G. (1996). Estrous and sex variations in vocalization thresholds to hindpaw and tail pressure stimulation in the rat. Brain Res 742: 352-354.

Keay KA, Bandler R (2001). Parallel circuits mediating distinct emotional coping reactions to different types of stress. Neurosci Biobehav Rev 25: 669-678.

Kincheski GC, Carobrez AP (2009). The dorsal periaqueductal gray modulates the increased fear-like behavior exhibited by experienced rats in the elevated plus maze. Behav Brain Res doi: 10.1016/j.bbr.2009.09.001.

Lautenbacher S, Spernal J, Schreiber W, Krieg JC (1999). Relationship between clinical pain complaints and pain sensitivity in patients with depression and panic disorder. Psychosomatic Medicine 61: 822-827.

Lovick TA (1996). Midbrain and medullary regulation of defensive cardiovascular functions. Prog Brain Res 107: 301-313.

Lovick TA, Griffiths JL, Dunn SM, Martin IL (2005). Changes in GABA(A) receptor subunit expression in the midbrain during the oestrous cycle in Wistar rats. Neuroscience 131: 397-405.

Martínez-Gómez M, Cruz Y, Salas M, Hudson R, Pacheco P (1994). Assessing pain threshold in the rat: changes with estrus and time of day. Physiol Behav 55: 651-657.

Mogil JS, Chesler EJ, Wilson SG, Juraska JM, Sternberg WF (2000). Sex differences in thermal nociception and morphine antinociception in rodents depend on genotype. Neurosci Biobehav Rev 24: 375-389.

Morgan MM, Whittier KL, Hegarty DM, Aicher SA (2008). Periaqueductal gray neurons project to spinally projecting GABAergic neurons in the rostral ventromedial medulla. Pain 140: 376-386.

Paxinos G, Watson C (2004). The Rat Brain in Stereotaxic Coordinates ( ${ }^{\text {th }}$ Edition). Academic Press: New York, USA.

Powell-Boone T, Ness TJ, Cannon R, Lloyd LK, Weigent DA, Fillingim RB (2005). Menstrual cycle affects bladder pain sensation in subjects with interstitial cystitis. J Urol 174: 1832-1836.

Purdy RH, Moore Jr PH, Rao PN, Hagino N, Yamaguchi T, Schmidt $\mathrm{P}$ et al. (1990). Radioimmunoassay of 3 alpha-hydroxy5 alpha-pregnan-20-one in rat and human plasma. Steroids 55: 290-296.

Rhudy JL, Meagher MM (2000). Fear and anxiety: divergent effects on human pain thresholds. Pain 84: 65-75.

Riley III J, Robinson ME, Wise EA, Price DD (1999). A metaanalytic review of pain perception across the menstrual cycle. Pain 81: 225-235.

Rizvi TA, Ennis M, Behbehani MM, Shipley MT (1991). Connections between the central nucleus of the amygdala and the midbrain periaqueductal gray: topography and reciprocity. J Comp Neurol 303: 121-131.

Rizzi A, Marzola G, Bigoni R, Guerrini R, Salvadori S, Mogil JS et al. (2001). Endogenous nociceptin signaling and stressinduced analgesia. Neuroreport 12: 3009-3013.

Rocher C, Spedding M, Munoz C, Jay TM (2004). Acute stressinduced changes in hippocampal/prefrontal circuits in rats: effects of antidepressants. Cerebral Cortex 14: 224-229. 
Ryan SM, Maier SF (1988). The estrous cycle and estrogen modulate stress-induced analgesia. Behav Neurosci 102: 371-380.

Salchner P, Singewald N (2002). Neuroanatomical substrates involved in the anxiogenic-like effect of acute fluoxetine treatment. Neuropharm 43: 1238-1248.

Shansky RM, Glavis-Bloom C, Lerman D, McRae P, Benson C, Miller $\mathrm{K}$ et al. (2004). Estrogen mediates sex differences in stress-induced prefrontal cortex dysfunction. Mol Psychiatry 9: 531-538.

Steiner M (1997). Premenstrual syndromes. Annu Rev Med 48: 447-455.

Taenzer P, Melzack R, Jeans ME (1986). Influence of psychological factors on postoperative pain, mood and analgesic requirements. Pain 24: 331-342.

Terner JM, Lomas LM, Picker MJ (2005). Influence of estrous cycle and gonadal hormone depletion on nociception and opioid antinociception in female rats of four strains. J Pain 6: 372-383.

Tousignant-Laflamme Y, Marchand S (2009). Excitatory and inhibitory pain mechanisms during the menstrual cycle in healthy women. Pain 146: 47-55.

Vendruscolo LF, Pamplona FA, Takahashi RN (2004). Strain and sex differences in the expression of nociceptive behavior and stress-induced analgesia in rats. Brain Res 1030: 277-283.

Vidal C, Jacob J (1982). Hyperalgesia induced by non-noxious stress in the rat. Neurosci Lett 32: 75-80.
Vinogradova EP, Zhukov DA, Batuev AS (2003). The effects of stages of the estrous cycle on pain thresholds in female white rats. Neurosci Behav Physiol 33: 69-72.

Walf AA, Frye CA (2003). Anti-nociception following exposure to trimethylthiazoline, peripheral or intra-amygdala estrogen and/ or progesterone. Behav Brain Res 144: 77-85.

Weinberg MS, Bhatt AP, Girotti M, Masini CV, Day HE, Campeau S et al. (2009). Repeated ferret odor exposure induces different temporal patterns of same-stressor habituation and novelstressor sensitization in both hypothalamic-pituitary-adrenal axis activity and forebrain c-fos expression in the rat. Endocrinology 150: 749-761.

Williams S, Evan GI, Hunt SP (1990). Changing patterns of c-fos induction in spinal neurons following thermal cutaneous stimulation in the rat. Neuroscience 36: 73-81.

You HJ, Cao DY, Yuan B, Arendt-Nielsen L (2006). Sex differences in the responses of spinal wide-dynamic range neurons to subcutaneous formalin and in the effects of different frequencies of conditioning electrical stimulation. Neuroscience 138: 1299-1307.

Zhang YQ, Tang JS, Yuan B, Jia H (1997). Inhibitory effects of electrically evoked activation of ventrolateral orbital cortex on the tail-flick reflex are mediated by periaqueductal gray in rats. Pain 72: 127-135. 